\title{
New Synthesis: Chemical Ecology and Sustainable Food Production
}

\section{J. A. Pickett}

(C) Springer Science+Business Media, LLC 2012

Chemical ecology as an intrinsically multidisciplinary subject must be vastly expanded to provide new technologies for sustainable food production in the face of global population rise and climate change. No longer can we roll out the unsubstantiated claim that the science is in place and that all we need to do is to transfer the technology. Not even the rapid advances in molecular genetics and particularly the dramatically increased access to complete genomic sequences and associated informatics are sufficient. They would benefit greatly from the potentially holistic approach offered by chemical ecology. Annotation of genomes currently is the step limiting progress, and chemical ecology is ideally suited to assigning biological function to genes associated with biosynthesis of plant secondary metabolites (PSMs). I have been attempting to bring about a new public understanding of the need for further development of genetically modified organisms (GMOs) for food production, and have encountered from both the scientific community and the public media many naïve questions relating to what the questioner believes are issues that we have overlooked. Chemical ecology encompasses these issues of multitrophic interactions and unexpected impacts of highly active metabolites, as is exemplified in the rules for publication in this Journal.

This should not imply that all chemical ecologically based approaches are the most sustainable. However, Baulcombe, in the report that he led on producing for the senior scientific academy of the UK (Royal Society, 2009), outlined the technologies necessary for sustainable intensification of food production, and many of these objectives can be tackled by chemical ecology. Recently, Phalan et al. (2011) provided evidence that sparing is more viable in this way than sharing. Thus, it is towards intensification that chemical ecology should primarily be directed and not in trying to create systems where agricultural production shares the same land in an attempt to maintain or expand species diversity. Specifically, to reduce reliance on seasonal inputs such as fertilizer, seed, pesticides and the energy needed to deliver these, in addition to the cost of soil preparation, more effects must be delivered via the seed, and eventually there needs to be a large scale movement towards perennial crops, including replacement of the current arable staples. Crop losses to pests, diseases, and weeds harm the environment as well as farm profitability because the carbon footprint of bringing forth the crop, from which there would be loss, has already been created. Therefore, powerful, integrated new approaches to providing plant nutrients and pest control are needed. The tools for many of these approaches will be PSMs studied and developed in the context of chemical ecology. Development can be by breeding but with newly available advances, such as alien introgression, that enable introduction of more potent traits from ancestral plants. Probably the most powerful approach will be via GMOs. For example, exploitation of defense benzoxazinoids in cereals such as rye, maize, and wheat, mostly lost from modern varieties and hybrids during

\section{J. A. Pickett $(\bowtie)$}

Rothamsted Research,

Harpenden, Hertfordshire AL5 2JQ, UK

e-mail: john.pickett@rothamsted.ac.uk breeding, could be enhanced or reintroduced, potentially providing control against pests and probably disease and weeds. By introducing nitrogen fixation into a wider range of crops, or into their rhizospheres, we can expect to harness allelopathy to reduce losses of fixed nitrogen as the powerful noxious greenhouse gas, nitrous oxide. Animal husbandry will also benefit in terms of new PSM-based pest control and use of molecular markers for animal breeding. Those geographic regions that support only grasslands for meat and dairy production could be made more acceptable by regulating the production of methane, the other major agrogenic greenhouse gas, by allelopathic traits in the grassland flora. Potential disadvantages and nontarget effects will be studied as part of the development process.

Management of gene expression can be achieved by naturally occurring elicitors such as the development of cis-jasmone. Opportunities will involve not just other elicitors that can naturally penetrate the plant cuticle and effect systemic upregulation of defense genes, but will give rise to a new way of activating useful genetic traits by the development of sentinel plants. Such sensitive plants detect problems, not just pests, diseases, and weed competition but also depleted or excess nutrients and water, and signal to the main crop of smart plants; the natural response to the signals comprising small lipophilic molecules linked to gene expression (by GM). Perennial plants, as pointed out in The Royal Society report (2009) will be the ultimate goal, and sentinel plants will be a means of managing the necessary genetics for plant traits without the need for passing through the crop with energy intensive implements.

Baulcombe called for vastly increased expenditure in research and in education. The various disciplines needed to provide the new science must be closely linked, and chemical ecology should provide the overall framework. Both high science and applied and agricultural work are necessary. Functional genes causing useful biological effects need to be identified and expression ensured by elicitors where necessary. Bioassay (i.e. phenotyping) guided fractionation, the underpinning approach of chemical ecology for identifying the new crop management tools, is too often ignored. Many studies merely involve trivial perturbation of natural physiology by randomly adding, often very weakly active, natural products in high physiological excess. We must stop doing this and not give plausibility to those that do. We must show the way through high quality science and thereby persuade the world of the contribution that chemical ecology can make to sustainable intensification of food production.

\section{References}

Phalan, B., Onial, M., Balmford, A., and Green, R. E. 2011. Reconciling food production and biodiversity conservation: Land sharing and land sparing compared. Science 333:1289-1291.

ROYAL SOCIETY. 2009. Reaping the benefits: science and the sustainable intensification of global agriculture. RS Policy document 11/09, 72 pp. (http://royalsociety.org/Reapingthebenefits/). 